\title{
SÍNTESIS DE NUEVOS COPOLÍMEROS EN BLOQUE A PARTIR DE POLIETILENGLICOL Y 2-OXAZOLINAS
}

\author{
Juan C. Rueda ${ }^{* a}$, Miguel Asmad ${ }^{\mathrm{a}}$, Marisol Sedano ${ }^{\mathrm{a}}$, Stefan Zschoche ${ }^{\mathrm{b}}$, \\ Brigitte Voit ${ }^{\mathrm{b}}$
}

\begin{abstract}
RESUMEN
Fueron obtenidos nuevos copolímeros en bloque (tipo AB), a partir de la polimerización catiónica por apertura de anillo de los monómeros 2-ciclopropil- y 2-metoxicarboniletil2-oxazolina, utilizando el polietilenglicol tosilado como iniciador. En los copolímeros en bloque, el grado de polimerización del segmento de polietilenglicol (PEG) fue siempre de 45 unidades y el de las poli(2-oxazolinas) fue variable, entre 33 y 242 unidades. Los copolímeros en bloque que contenían 2-metoxicarboniletil-2-oxazolina, fueron sometidos a una hidrólisis de sus grupos éster para obtener cuantitativamente grupos ácido carboxílicos. Los copolímeros fueron caracterizados estructuralmente mediante resonancia magnética nuclear y también mostraron una transición conformacional (LCST) entre 24 y $48^{\circ} \mathrm{C}$, que fue función de la estructura del polímero, de su contenido de ciclopropiloxazolina y de los grupos ácido carboxílicos.
\end{abstract}

Palabras clave: Copolímeros, poli(etilenglicol), poli(2-oxazolinas).

\section{SYNTHESIS OF NEW BLOCK COPOLYMERS BASED ON POLYETHYLENGLYCOL AND 2-OXAZOLINES}

\begin{abstract}
New block copolymers (typ AB) were obtained by the ring opening cationic polymerization of 2-cyclopropyl- and 2-methoxycarbonylethyl-2-oxazoline, using tosylated polyethylenglycol as initiator. In block copolymers, the polymerization grade of polyethylenglycol (PEG) segment was always 45 units and of the poly(2-oxazolines) segment was variable, between 33 to 242 units. The block copolymers, containing 2-methoxycarbonylethyl-2-oxazoline, were submitted to a quantitative hydrólisis of ester groups to obtain carboxylic acid units. The copolymers were characterized structurally by nuclear magnetic resonance spectroscopy and they show a conformational transition (LCST) between 24 and $48^{\circ} \mathrm{C}$, which was function of the polymer structure, of its content of cyclopropyloxazoline and carboxilic acid groups.
\end{abstract}

Keywords: copolymers, poly(ethylenglycol), poly(2-oxazolines).

\section{INTRODUCCIÓN}

Actualmente uno de los principales temas de investigación en la química de los polímeros es

\footnotetext{
a) Pontificia Universidad Católica del Perú, Dirección de Gestión de la Investigación, Sección Física, Lab. de Polímeros, Av. Universitaria 1801, San Miguel, Lima, jrueda@pucp.edu.pe

b) Instituto Leibniz de Investigaciones en Polímeros de Dresden, Alemania.
} 
la búsqueda de polímeros con propiedades específicas que puedan solucionar determinados problemas tecnológicos en campos tan diversos como, por ejemplo, la biotecnología, la medicina, la electrónica, o la óptica. Es interesante, por ejemplo, la síntesis de polímeros para ser aplicados en la elaboración de nanomateriales, en sistemas de liberación controlada de medicamentos o de fertilizantes, en el control reológico, en la elaboración de membranas, películas delgadas, y sensores, entre otros. Los polímeros que presentan una respuesta o sensibilidad a variaciones de parámetros ambientales tales como, por ejemplo, la temperatura o el grado de acidez del medio $(\mathrm{pH})$, son denominados polímeros inteligentes (smart polymers en inglés) y son una interesante alternativa de solución ${ }^{1-3}$. La respuesta o sensibilidad térmica que tienen ciertos polímeros, cuando están en solución acuosa, se expresa en forma de una transición conformacional. A una determinada temperatura (denominada en ingles Low Critical Solution Temperature-LCST), el polímero pasa de una estructura extendida (e hidrofílica) a una estructura globular colapsada (e hidrofóbica). Dentro del grupo de polímeros que presentan esta sensibilidad térmica se encuentran, entre otros, la poli( $\mathrm{N}$-isopropilacrilamida), poli(ciclopropiloxazolina), polietiloxazolina, y sus respectivos copolímeros.

Por otro lado, los materiales que presentan una respuesta al $\mathrm{pH}$ son polímeros que, por ejemplo, contienen en su estructura grupos funcionales tipo ácido carboxílicos o aminas ${ }^{4,5}$.

La N-isopropilacrilamida polimeriza vía radicales libres y debido a que en este tipo de polimerización ocurren siempre reacciones secundarias, tales como, reacciones de terminación o de transferencia de cadena, no es posible controlar en forma precisa el peso molecular de los polímeros obtenidos ni la funcionalidad de los mismos.

Una variante muy interesante a explorar es la síntesis de polímeros con estructuras y funcionalidades predeterminadas en base a monómeros de 2-oxazolinas aprovechando la polimerización controlada (polimerización catiónica "viva") que ocurre con este tipo de monómeros. De tal manera que es posible controlar con precisión tanto el peso molecular y la distribución del mismo así como la funcionalidad de las cadenas poliméricas ${ }^{6-8}$.

El polietilenglicol (PEG) por su lado, es un polímero soluble en agua, barato, biocompatible, y fácilmente modificable químicamente ${ }^{9}$.

En la presente investigación hemos estudiado la factibilidad de la síntesis de copolímeros en bloque, sensibles a la temperatura y al $\mathrm{pH}$, a partir de PEG y 2-oxazolinas.

\section{PARTE EXPERIMENTAL}

Las sustancias químicas utilizadas en esta investigación fueron obtenidas de las firmas Aldrich, Fluka y Merck y fueron purificadas mediante métodos estándar descritos en la literatura ${ }^{4,7,10}$. Los monómeros 2-ciclopropil-2-oxazolina (ciclopoxa) y 2-metoxicarboniletil-2-oxazolina (esteroxa) fueron sintetizados mediante los procedimientos descritos en la bibliografía ${ }^{10-14}$.

Tosilación del polietilenglicol (PEG-Ts): El polietilenglicol (PEG de grado de polimerización 
45 y dispersión 1,5, de Fluka) fue tosilado mediante el siguiente procedimiento: 6,04 g (3 mmol) de PEG y 1,52 g (15 mmol) de trietilamina fueron disueltos en $12 \mathrm{ml}$ de cloruro de metileno. A esta solución se le adicionó, gota a gota, una solución de cloruro de tosilo $(1,40 \mathrm{~g}$, $7,35 \mathrm{mmol}$ ) en $10 \mathrm{~mL}$ de cloruro de metileno y se agitó por 12 horas a $-10^{\circ} \mathrm{C}$. La solución fue concentrada mediante el evaporador rotatorio y luego precipitada en éter dietílico. Después de la filtración, el producto fue disuelto en tetrahidrofurano para remover las sales. La fase orgánica fue evaporada y secada en vacío. Rendimiento 6,3 g (96\%).

RMN del PEG: ${ }^{1} \mathrm{H}-\mathrm{RMN}\left(\mathrm{en}_{\mathrm{CDCl}}\right) \delta: 3,4\left(\mathrm{OCH}_{3}\right) ; 3,5-3,7\left(\mathrm{OCH}_{2} \mathrm{CH}_{2}\right)$. ${ }^{13} \mathbf{C}-\mathbf{R M N}\left(\mathbf{e n ~} \mathbf{C D C l}_{3}\right) \boldsymbol{\delta}: 58\left(\mathrm{OCH}_{3}\right) ; 60-72\left(\mathrm{OCH}_{2} \mathrm{CH}_{2}\right)$.

RMN del PEG-Tosilado (ver discusión de resultados)

Equipo RMN: Brucker, frecuencias $250 \mathrm{MHz}$ para protón y $125 \mathrm{MHz}$ para carbono 13. Solventes: cloroformo y agua deuterados.

Síntesis de los copolímeros en bloque: Procedimiento típico: En un balón de $50 \mathrm{ml}$, con entrada lateral de nitrógeno, se disolvieron $0,30 \mathrm{~g}$. $\left(1,39 \times 10^{-4}\right.$ moles $)$ de polietilenglicol tosilado (PEG-Ts) en 4,0 ml de benzonitrilo. Luego se añadió a esta solución 0,607 gr. $\left(5,46 \times 10^{-3}\right.$ moles $)$ de 2-ciclopropiloxazolina (en otros casos se añadió la 2-metoxicarboniletil2-oxazolina o la mezcla de ambos monómeros). Se cerró el balón de reacción bajo atmósfera de nitrógeno secó y se mantuvo a una temperatura de $55^{\circ} \mathrm{C}$ por 72 horas, con agitación constante. Transcurrido este tiempo de reacción, se dejó enfriar el balón lentamente hasta la temperatura ambiente y se añadió una solución de $\mathrm{KOH}\left(7,9 \times 10^{-3} \mathrm{~g}, 1,4 \times 10^{-4}\right.$ moles $)$ en metanol para terminar la polimerización.

Se procedió a purificar el polímero obtenido mediante el método de precipitación. Para esto, en primer lugar, se eliminó la mayor parte del benzonitrilo por evaporación y luego se disolvió el resto en $10 \mathrm{ml}$ de cloroformo y se añadió lentamente esta solución a $120 \mathrm{~mL}$ de heptano, bajo agitación. El polímero precipitó inmediatamente. Se filtró y se secó el polímero obtenido a una temperatura de $36^{\circ} \mathrm{C}$, bajo vacío. Se volvió a repetir este procedimiento de precipitación dos veces más. Los polímeros fueron caracterizados estructuralmente mediante sus espectros de resonancia magnética nuclear.

Ejemplo típico: MSP2: ${ }^{1} \mathbf{H}-\mathbf{R M N}\left(\mathbf{e n} \mathbf{C D C l}_{3}\right) \delta$ : 0,70 $\left(\mathrm{CH}_{2} \mathrm{CH}_{2}\right.$ (ciclopoxa)); 1,60 (CH (ciclopoxa)); 3,0-3,9 $\left(\mathrm{NCH}_{2} \mathrm{CH}_{2}\right)$; 3,5-3,7 (señales del grupo OCH2CH2 del PEG que están traslapadas con las señales de $\mathrm{NCH} 2 \mathrm{CH} 2$ ).

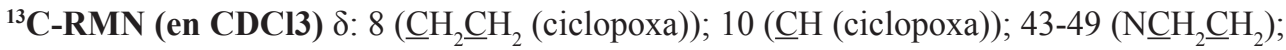
$70\left(\mathrm{OCH}_{2} \underline{\mathrm{CH}}_{2}\right.$ del $\left.\mathrm{PEG}\right) ; 174$ (carbonilo $\mathrm{C}=\mathrm{O}$ de la polioxazolina).

Hidrólisis de los copolímeros en bloque: Ejemplo: se disolvió 1,0 g. del polímero MSP4, en $20 \mathrm{ml}$. de una mezcla de $10 \mathrm{ml}$ de una solución de hidróxido de sodio $0,1 \mathrm{~N}$ y $10 \mathrm{ml}$. de metanol. La mezcla fue calentada a $45^{\circ} \mathrm{C}$ durante 7 horas, con agitación constante. Terminado este periodo se dejó enfriar la mezcla hasta temperatura ambiente y se acidificó la solución con $\mathrm{HCl} 0,1 \mathrm{~N}$ hasta obtener un $\mathrm{pH}=5,5$. Finalmente, el metanol y parte del agua fueron 
eliminados en un evaporador rotatorio. $\mathrm{El} \mathrm{NaCl}$ formado en la reacción y disuelto en la solución acuosa polimérica fue eliminado mediante una membrana de diálisis (Cut off $=1000$ ). Luego el polímero fue obtenido por liofilización. Se obtuvo un copolímero de color blanco al que se lo denominó H-MSP4 y fue caracterizado estructuralmente por resonancia magnética nuclear $(\mathrm{RMN})$.

${ }^{1} \mathbf{H}-\mathbf{R M N}$ (en $\left.\mathbf{D}_{2} \mathbf{O}\right) \quad \delta: 0,6 \quad\left(\mathrm{CH}_{2} \mathrm{CH}_{2}, \quad\right.$ (ciclopoxa)); 1,7 (CH, (ciclopoxa)); 2,2-2,6 $\left(\mathrm{COCH}_{2} \mathrm{CH}_{2} \mathrm{CO}\right.$, (esteroxa)); 3,0-3,9 $\left(\mathrm{NCH}_{2} \mathrm{CH}_{2}\right.$ (ciclopoxa+esteroxa)); 3,5-3,7 $\left(\mathrm{OCH}_{2} \mathrm{CH}_{2}\right.$ del PEG).

${ }^{13} \mathbf{C}-\mathbf{R M N}\left(\mathbf{e n} \mathbf{D}_{2} \mathbf{O}\right) \delta: 8\left(\underline{\mathrm{CH}}_{2} \underline{\mathrm{C}} \mathrm{H}_{2}\right.$ (ciclopoxa)); 10 (ㄷH (ciclopoxa)); 28, $32\left(\underline{\mathrm{COC}} \mathrm{H}_{2} \underline{\mathrm{C}} \mathrm{H}_{2} \underline{\mathrm{CO}}\right.$, esteroxa); 43-49 ( $\underline{\mathrm{C}}_{2} \underline{\mathrm{CH}}_{2}$ (ciclopoxa y esteroxa)); $175-177$ (señales de $\underline{\mathrm{C}}=\mathrm{O}$ y $\underline{\mathrm{COOH}} \mathrm{Oe}$ ciclopoxa y esteroxa).

\section{Determinación de la temperatura de transición conformacional (LCST) de los copolímeros en bloque}

Procedimiento típico: Se disolvió 0,050 g. de polímero H-MSP4 en $5 \mathrm{ml}$. de agua destilada, para conseguir obtener una concentración de polímero al 1\% en peso. Luego se filtró la solución y se colocó ésta en una cubeta del espectrómetro UV/VIS (Helios Gamma, Thermoelectron Corp.). Se colocó una termocupla flexible dentro de la cubeta, de tal manera que se pudiera medir en forma directa y a cada instante, la temperatura de la solución polimérica contenida en la cubeta al mismo tiempo que se medía la transmitancia. La temperatura de la cubeta fue variada con la ayuda de una chaqueta de calentamiento y de un baño termostatizado. Con el espectrómetro UV/Vis se procedió a medir el porcentaje de transmitancia $(\% \mathrm{~T})$ versus la temperatura $(\mathrm{T})$ de la solución polimérica a una longitud de onda constante de $550 \mathrm{~nm}$. Al inicio de la medición, a bajas temperaturas, cuando la solución polimérica es transparente, el porcentaje de transmitancia es de aproximadamente $100 \%$ y a medida que la temperatura va aumentando, el valor de la transmitancia comienza a disminuir, debido a la precipitación del polímero en el agua (fenómeno LCST). En los gráficos se tomó como valor de la temperatura de transición conformacional (LCST) el punto de inflexión de la curva.

\section{RESULTADOS Y DISCUSIÓN}

Tosilación del polietilenglicol: El polietilenglicol (PEG) fue modificado mediante la reacción del terminal hidroxilo del PEG con el cloruro de tosilo en medio etéreo y en presencia de trietilamina9 (esquema 1). El PEG usado tuvo un peso molecular de $2000 \mathrm{y}$ grado de polimerización 45 (Fluka) y solo en un extremo tuvo un grupo hidroxilo ya que en el otro tuvo un grupo metoxilo inerte. El PEG tosilado fue caracterizado vía RMN (figura 1):

${ }^{1} \mathbf{H}-\mathbf{R M N}\left(\mathbf{e n ~} \mathbf{C D C l}_{3}\right) \delta: 3,4\left(\mathrm{OCH}_{3}\right) ; 3,5-3,7\left(\mathrm{OCH}_{2} \mathrm{CH}_{2}\right) ; 4,1\left(\mathrm{OCH}_{2} \mathrm{CH}_{2}-\mathrm{Ts}\right) ; 7,3$ y 7,8 (protones aromáticos provenientes del grupo tosilo).

${ }^{13} \mathbf{C}-\mathbf{R M N}(\mathbf{e n} \mathbf{C D C l})$ ) $58\left(\mathrm{OCH}_{3}\right) ; 60-72\left(\mathrm{OCH}_{2} \mathrm{CH}_{2}\right) ; 127-130$ (protones aromáticos del grupo tosilo). 

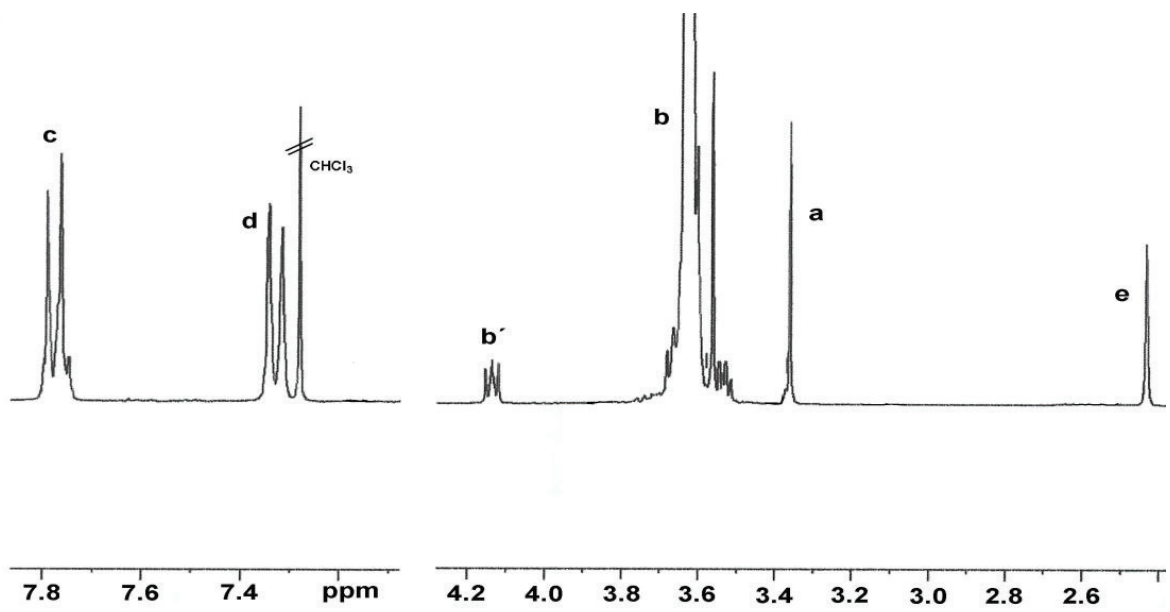

Figura 1. Espectro ${ }^{1} \mathrm{H}-\mathrm{RMN}$ del polietilenglicol tosilado en cloroformo deuterado a $25^{\circ} \mathrm{C}$ (ver la asignación de señales en el esquema 1 ).

En el espectro ${ }^{1} \mathrm{H}-\mathrm{RMN}$ del PEG-Ts se determinó que el final de cadena del PEG fue tosilado cuantitativamente (al 100\%) porque al comparar las integrales corregidas de los protones aromáticos del grupo tosilo y del mero del PEG; éstas estuvieron en la proporción teórica de 1 a 45.

Por otro lado, fue demostrado experimentalmente que el PEG es sensible a temperaturas relativamente altas, por lo que en las reacciones de polimerización siempre fueron usadas temperaturas bajas $\left(<56^{\circ} \mathrm{C}\right)$ para evitar la degradación del PEG.

\section{Síntesis de los copolímeros en bloque}

La síntesis de los copolímeros en bloque fue llevada a cabo mediante la polimerización catiónica por apertura de anillo de las 2-oxazolinas (ciclopoxa, esteroxa) iniciada por el polietilenglicol tosilado (PEG-Ts). El PEG-Ts funcionó como un macroiniciador en esta polimerización. Es conocido de la literatura que los ésteres tosilo son efectivos iniciadores de la polimerización de las 2-oxazolinas ${ }^{6,9}$. 

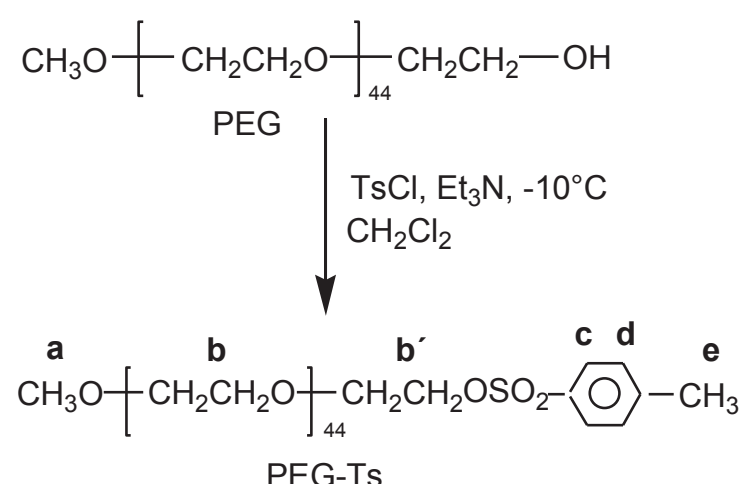

PEG-Ts
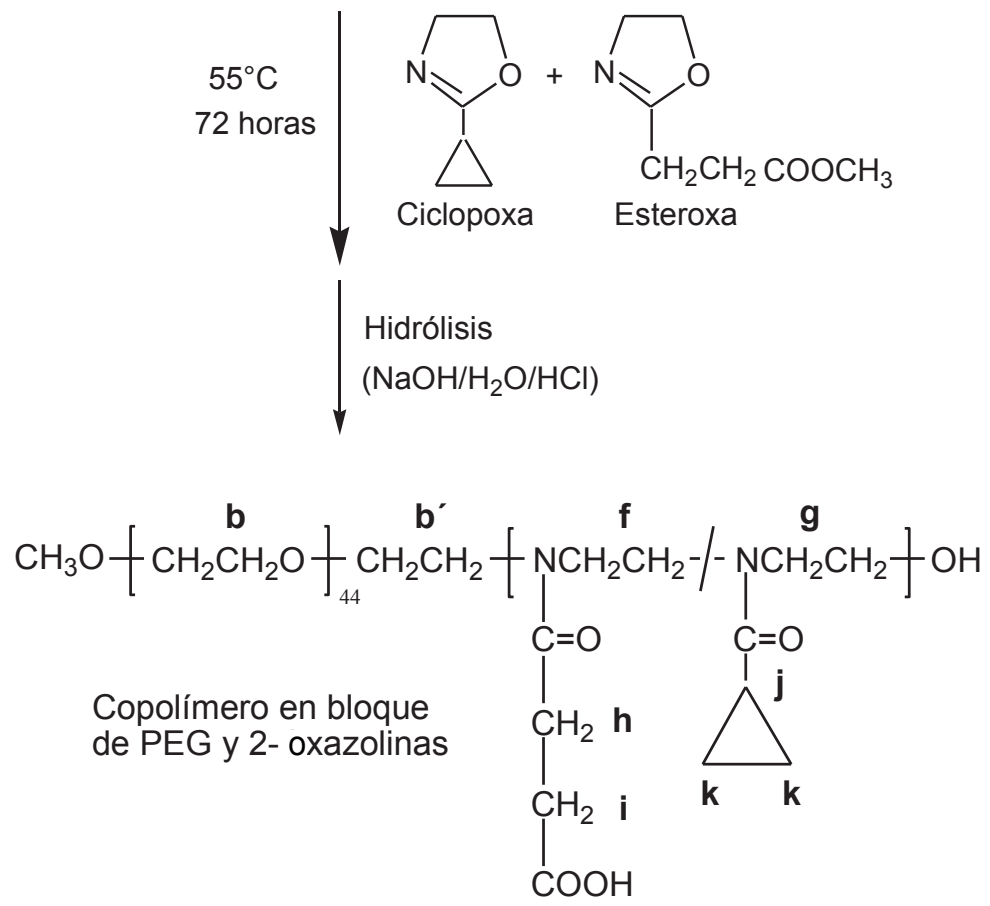

Esquema 1. Tosilación del PEG y subsecuente polimerización de las 2-oxazolinas mediante el PEG tosilado. 
El mecanismo de polimerización propuesto es el siguiente: los monómeros esteroxa o ciclopoxa realizan un ataque nucleofílico al grupo metileno del PEG-Ts que es adyacente al grupo tosilo y producen de esta forma un catión oxazolínico y el contraión tosilato. Este catión oxazolínico es a su vez atacado por una nueva molécula de monómero, abriéndose el anillo produciendo un polímero lineal con un nuevo catión oxazolínico en el final de cadena. Este proceso se repite muchas veces formándose entonces un segmento polimérico de 2-oxazolina unido al segmento de PEG. Finalmente, las cadenas crecientes son terminadas por la reacción del catión oxazolínico y los grupos hidroxilo adicionados $(\mathrm{KOH})$.

De esta manera se obtuvo una polimerización de tipo iónico formándose copolímeros en bloque de PEG y las 2-oxazolinas (esquema 1). La polimerización fue realizada a baja temperatura debido a que el PEG es susceptible de sufrir una degradación a altas temperaturas; por esto se trabajó siempre a $55^{\circ} \mathrm{C}$ y debido a que a esta temperatura la velocidad de reacción es baja se tuvo que emplear largos tiempos de reacción, de 72 horas, para conseguir la polimerización. En la tabla 1 se presenta las condiciones usadas y los resultados obtenidos.

Tabla 1. Síntesis de Copolímeros en Bloque mediante la polimerización de 2-ciclopropil- y 2-metoxicarboniletil-2-oxazolina, iniciada por el PEG-Ts ${ }^{\mathrm{a}}$

\begin{tabular}{lcccc}
\hline Polímero $^{\mathrm{b}}$ & \multicolumn{2}{c}{ Esteroxa/PEG-Ts $^{\mathrm{c}}$} & \multicolumn{2}{c}{ Ciclopoxa/PEG-Ts $^{\mathrm{d}}$} \\
& Teórico & Experimental & Teórico & Experimental \\
\hline MSP1 & 40 & 33 & -- & -- \\
MSP2 & -- & -- & 39 & 35 \\
MSP3 & -- & -- & 260 & 242 \\
MSP4 & 12 & 8 & 130 & 120 \\
MSP5 & 100 & 90 & -- & -- \\
& & & & \\
\hline
\end{tabular}

a) Las polimerizaciones se realizaron en benzonitrilo $(4 \mathrm{ml})$, a $55^{\circ} \mathrm{C}$ y en 72 horas (en el caso de MSP3 se usó 120 horas). Se usó en todos los casos 0,30 g de PEG-Ts, de grado de polimerización n=45 y dispersión 1,5 (Fluka).

b) Copolímeros en bloque elaborados. Cuando los copolímeros fueron hidrolizados, para nombrarlos se colocó la letra $\mathrm{H}$ delante de su denominación.

c) y d) Razones molares Esteroxazolina/PEG-Ts y Ciclopropiloxazolina/PEG-Ts en el copolímero en bloque: teórico= relación molar al inicio de la polimerización; y experimental=relación molar al final de la polimerización, determinada vía análisis cuantitativo del espectro RMN.

e) Los rendimientos de la polimerización fueron mayores al $85 \%$. 
Los copolímeros en bloque, que contenían al monómero Esteroxa, se hidrolizaron en medio básico, en condiciones "suaves" ( $\mathrm{NaOH} 0,1 \mathrm{~N}, 50^{\circ} \mathrm{C}, 7$ horas) para hidrolizar sólo a los grupos ésteres y no a los grupos amida. Los copolímeros en bloque hidrolizados y no hidrolizados fueron caracterizados vía RMN. A modo de ejemplo se muestra en la figura 2 el espectro ${ }^{1} \mathrm{H}$ RMN del copolímero hidrolizado H-MSP4. Mediante el RMN se determinó que dentro de la estructura del copolímero existen los meros de esteroxa, ciclopropoxa y PEG y se halló el grado de polimerización de los segmentos de esteroxa y ciclopoxa haciendo el cálculo comparativo de las integrales de las señales de estos monómeros ( $\underline{\mathrm{f}}, \mathrm{g}, \underline{\mathrm{h}}, \underline{\mathrm{i}} \mathrm{y} \underline{\mathrm{k}}$ ) versus la integral de la señal de PEG (b $)$. Para el cálculo, se tomó en cuenta que en la región entre 3,0 y 4,0 ppm se tiene la suma de las señales b, f y g.

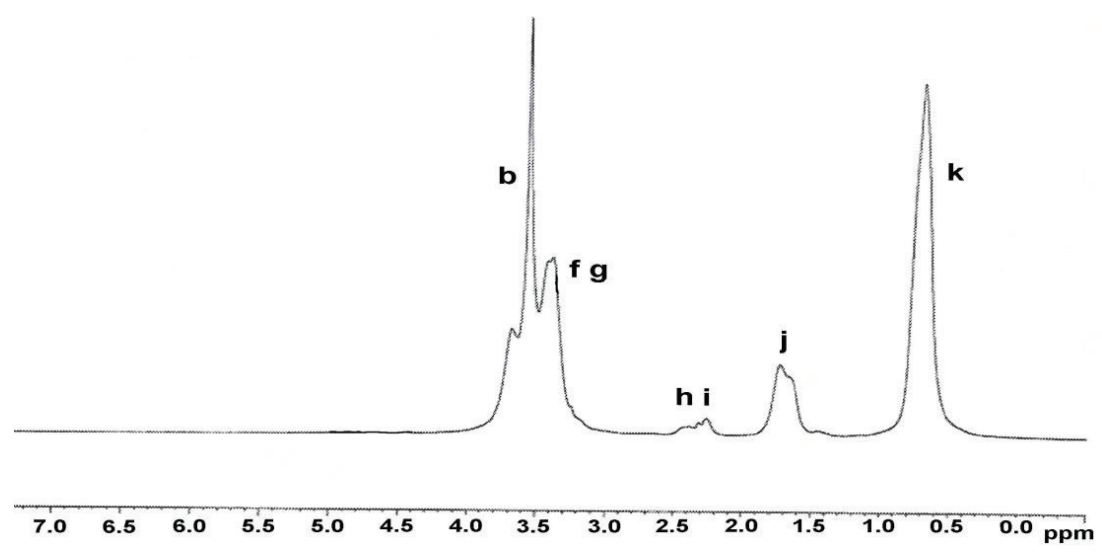

Figura 2. Espectro ${ }^{1} \mathrm{H}-\mathrm{RMN}$ del copolímero hidrolizado H-MSP4 en agua deuterada. La asignación de señales se realizó según el esquema 1.

\section{Determinación de la temperatura de transición conformacional (LCST) de los copolímeros en bloque}

Fueron realizadas determinaciones de la temperatura de transición conformacional (Low Critical Solution Temperature-LCST) de los copolímeros en bloque en soluciones acuosas para determinar su sensibilidad a la temperatura y al $\mathrm{pH}$. En todos los copolímeros el grado de polimerización de PEG fue el mismo $(n=45)$ por lo que las variaciones observadas se debieron exclusivamente al contenido relativo de los segmentos de policiclopoxa y poliesteroxa.

Se realizó mediciones turbidimétricas (ploteo del porcentaje de transmitancia versus la temperatura) utilizando el espectrómetro UV/Visible a una longitud de onda fija de $550 \mathrm{~nm}$.

En la figura 3 se muestra las curvas de transmitancia versus la temperatura para las soluciones acuosas al 1\% de las muestras MSP2 y MSP3, que contienen sólo PEG y ciclipropoxa. Se 
observa en primer lugar que ambos polímeros muestran una caída de la transmitancia en función de la temperatura, que es debida a la precipitación del polímero en el agua (fenómeno LCST) por el colapso inter- e intramolecular de los segmentos termosensibles de policiclopoxa dentro de los copolímeros en bloque.

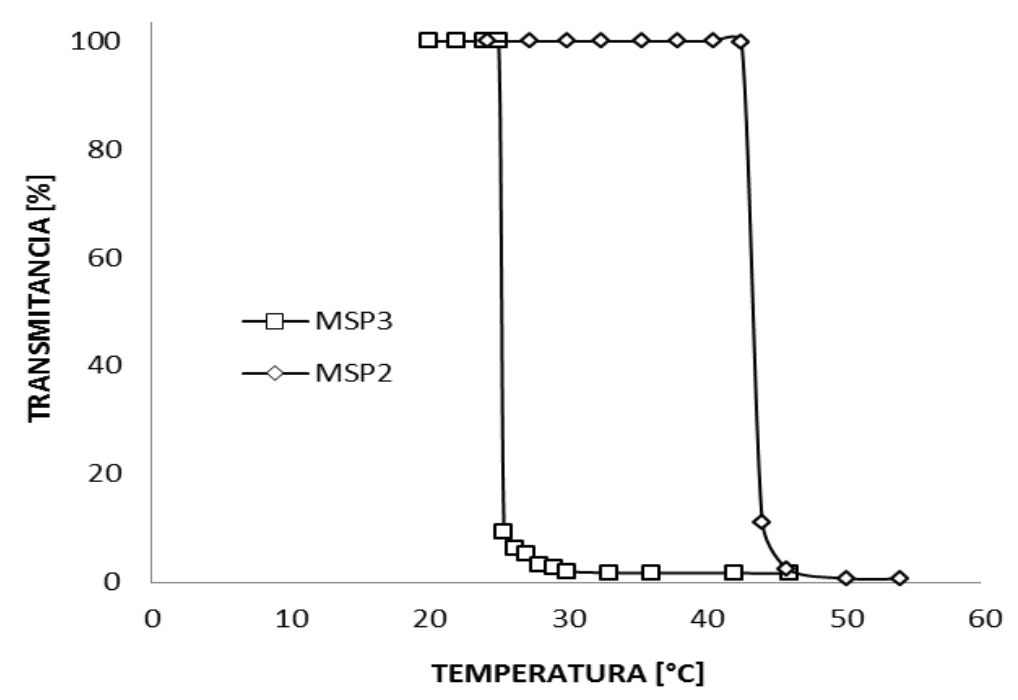

Figura 3. Determinación de LCST para la muestras MSP2 $\left(\mathrm{LCST}=42^{\circ} \mathrm{C}\right)$ y MSP3 $\left(\mathrm{LCST}=24^{\circ} \mathrm{C}\right)$.

Al comparar las curvas obtenidas para estos polímeros se observa que el MSP3 tiene un LCST más bajo que el MSP2 $\left(24\right.$ vs $\left.42^{\circ} \mathrm{C}\right)$. Esto es lógico de esperar ya que el MSP3 tiene una cadena hidrofóbica (policiclopoxa) más larga que MSP2 ( $\mathrm{n}=242$ vs 35) y debido a esta mayor hidrofobicidad ocurre con este polímero en primer lugar el colapso LCST en forma similar a lo que ocurre con el polímero termosensible poliN-isopropilacrilamida (poliNiPAAm) de diferentes pesos moleculares ${ }^{15}$. Eventualmente al formarse un núcleo hidrofóbico de policliclopoxa se podrían formar micelas o agregados moleculares con la cadena hidrofilica de PEG pero esto no ocurre talvez por ser esta cadena relativamente corta.

En la figura 4 se muestran las curvas de porcentaje de transmitancia versus temperatura para el copolímero MSP4 hidrolizado y no hidrolizado, H-MSP4 y MSP4, respectivamente. Se observa que el LCST aumenta al hidrolizarse el copolímero. Los grupos carboxilatos de sodio generados en la hidrólisis son fuertemente hidrofilicos y provocan un aumento del LCST. Es conocido de la literatura con polímeros similares, que el fenómeno LCST ocurre a temperaturas más elevadas a un mayor grado de hidrofilidad del polímero ${ }^{4,5,10}$. 


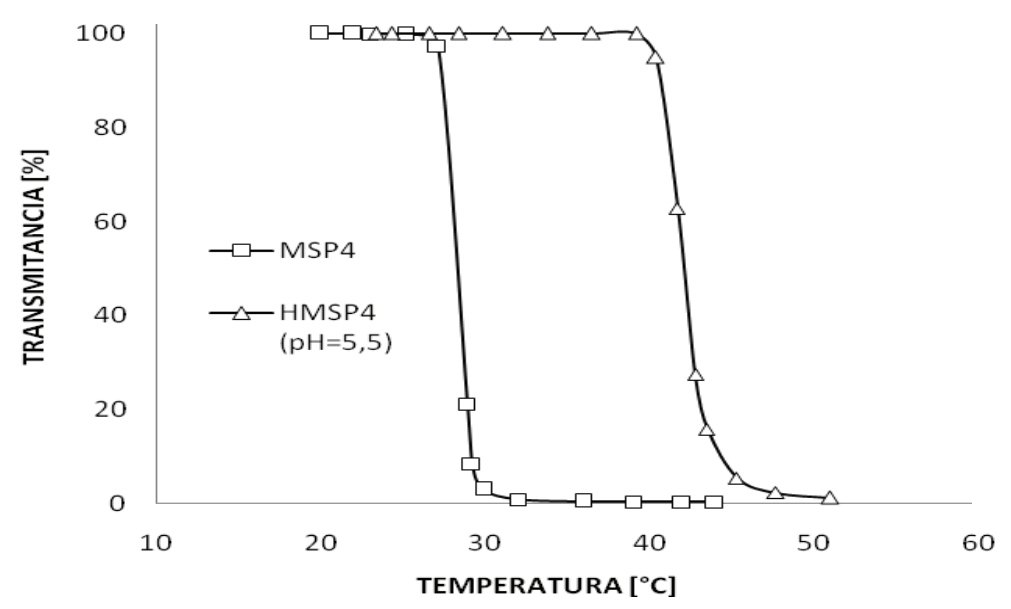

Figura 4. Determinación de LCST para la muestra MSP4 antes $\left(\mathrm{LCST}=28^{\circ} \mathrm{C}\right) \mathrm{y}$ después de la hidrólisis (H-MSP4, $\mathrm{pH} 5,5, \mathrm{LCST}=42^{\circ} \mathrm{C}$ ).

En la figura 5 se plotea el porcentaje de transmitancia versus la temperatura que produce el polímero hidrolizado H-MSP4 en solución acuosa a diferentes valores de $\mathrm{pH}$. Se observa que a un $\mathrm{pH}$ bajo, como 1, ocurre el LCST a menor temperatura y la curva es drásticamente vertical o sea el fenómeno acontece sumamente rápido, de forma intensa, y en un muy corto intervalo de temperatura.

Se hipotetiza, en forma similar a sistemas poliméricos similares ${ }^{5,7,14}$, que al producirse el LCST a este $\mathrm{pH}$, se podrían formar puentes de hidrógeno entre los grupos ácido carboxílicos y los grupos amida y esto unido al fenómeno LCST (colapso intra- e intermolecular de las macromoléculas), haría que colapsen rápidamente todas las macromoléculas juntas.

Por otro lado, a un $\mathrm{pH}$ alto como, por ejemplo, 10,5 todos los grupos ácido están ya como grupos carboxilatos de sodio, los cuales son fuertemente polares, y por esto al colapsar el segmento de policiclopropiloxazolina los grupos polares carboxilatos de sodio y los segmentos de PEG quedarían todavía parcialmente solvatados por las moléculas de agua debido a su hidrofilicidad y por esto harían que el fenómeno LCST ocurra a temperaturas más elevadas y con menor intensidad.

$\mathrm{A}$ un $\mathrm{pH}$ intermedio ocurre un efecto intermedio entre estas dos situaciones antes mencionadas y por eso el LCST tiene un valor intermedio. Los LCST para este polímero son 28,42 y $48^{\circ} \mathrm{C}$ a $\mathrm{pH}=1,5,5$ y 10,5 , respectivamente. 


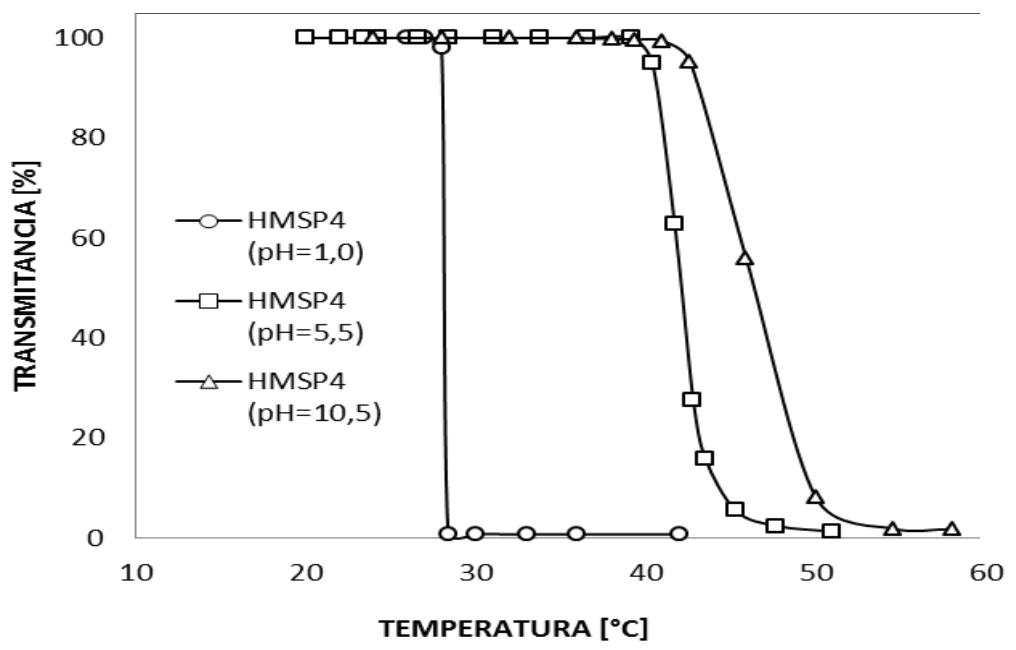

Figura 5. Determinación de LCST de la muestra H-MSP4 (muestra MSP4 hidrolizada) a diferentes valores de $\mathrm{pH}$.

\section{CONCLUSIONES}

Es posible la síntesis de polímeros sensibles a la temperatura y al $\mathrm{pH}$ mediante la polimerización de los monómeros 2-ciclopropil-2-oxazolina y 2-metoxicarboniletil-2-oxazolina iniciada por el polietilenglicol tosilado. Los copolímeros obtenidos fueron del tipo copolímero en bloque. Los copolímeros en bloque mostraron temperaturas de transición conformacional (LCST) que son función de la estructura del polímero, del contenido de 2-ciclopropil-2-oxazolina y de los grupos ácido carboxílicos dentro del polímero.

Estos nuevos materiales poliméricos podrían encontrar aplicación en los campos biotecnológico, de liberación controlada de medicamentos y en el de los sensores y actuadores.

\section{BIBLIOGRAFÍA}

1. Cohen M., Huck W., Genzer J., Mueller M., Ober C., et al.. Nature Materials, 2010; 9: 101-113.

2. Okano, T., Ed.; Biorelated Polymers and Gels; Academic Press: San Diego, CA, 1998.

3. Langer, R.; Tirrell, D. A., Nature, 2004; 428: 487-492.

4. Schild, H. G., Prog. Polym. Sci., 1992; 17: 163-249.

5. Dimitrov I., Trzebicka B., Müller A., Dworak A., Prog. Polym. Sci, 2007; 32: 1275 13436. $\quad$ Aoi, K.; Okada, M., Prog. Polym. Sci., 1996; 21: 151-208.

7. Rueda J., Zschoche S., Komber H., Schmaljohann D., Voit B., Macromolecules, 2005; 38: 7330-7336.

8. Rueda J., Zschoche S., Komber H., Krahl F., Arndt K-F., Voit B., Macromol. Chem. 
Phys., 2010; 211: 706-711.

9. Brissault B., Guis C., Cheradame H., European Polymer Journal, 2002; 38: 219-228.

10. Bloksma M., Schubert U., Hoogenboom R., Macromol Rapid Commun., 2011; 32: 1419-1441.

11. Witte H., Seeliger W., Liebigs Ann. Chem., 1974; 996-1009.

12. Zarka, M.; Nuyken, O.; Weberskirch R., Chem. European J., 2003; 9: 3228.

13. Levy, A.; Litt, M. H., J. Polym. Sci. Part A, 1968; 16: 1883.

14. Zschoche S., Rueda J. C., Binner M., Komber H., et. al., Macromol. Chem. Physic, 2011; 213 : 215-226.

15. Xia Y., Yin X., Burke A. D., Stöver H., Macromolecules, 2005; 38: 5937-5943. 